\title{
Multiplicative Schwartz-Type Block Multi-Color Gauss-Seidel Smoother for Algebraic Multigrid Methods
}

\author{
Masatoshi Kawai \\ RIKEN Center for Computational \\ Science \\ Kobe, Japan \\ masatoshi.kawai@riken.jp
}

\author{
Akihiro Ida \\ The University of Tokyo, \\ Information Technology Center \\ Tokyo, Japan
}

\author{
Hiroya Matsuba \\ RIKEN Center for Computational \\ Science \\ Kobe, Japan
}

\author{
Kengo Nakajima \\ The University of Tokyo, \\ Information Technology Center \\ Tokyo, Japan
}

\author{
Matthias Bolten \\ University of Wuppertal \\ Wuppertal, Germany
}

\begin{abstract}
In this paper, we propose a multiplicative Schwartz-type block multicolor Gauss-Seidel (MS-BMC-GS) smoother for algebraic multigrid (AMG) methods. AMG is an excellent solver and one of the most effective preconditioners for Krylov subspace methods such as the conjugate gradient method. The achievable degree of parallelism, convergence ratio, and computational cost of AMG strongly depend on the chosen smoother. As multiple unknowns are relaxed simultaneously, the MS-BMC-GS smoother realizes higher convergence than the existing parallel Gauss-Seidel smoother. Although this increases the amount of computation, the increase in the computational time is mitigated by the high cache hit ratio owing to the novel blocking technique. Numerical experiments demonstrate that MS-BMC-GS outperforms the block multi-color GS smoother by $18 \%$.
\end{abstract}

\section{CCS CONCEPTS}

- Mathematics of computing $\rightarrow$ Solvers.

\section{KEYWORDS}

Parallel computing, Multigrid solver, AMG, smoother, block multicoloring

\section{ACM Reference Format:}

Masatoshi Kawai, Akihiro Ida, Hiroya Matsuba, Kengo Nakajima, and Matthias Bolten. 2020. Multiplicative Schwartz-Type Block Multi-Color Gauss-Seidel Smoother for Algebraic Multigrid Methods. In International Conference on High Performance Computing in Asia-Pacific Region (HPCAsia2020), January 15-17, 2020, Fukuoka, Japan. ACM, New York, NY, USA, 10 pages. https://doi.org/10.1145/3368474.3368481

Permission to make digital or hard copies of all or part of this work for personal or classroom use is granted without fee provided that copies are not made or distributed for profit or commercial advantage and that copies bear this notice and the full citation on the first page. Copyrights for components of this work owned by others than ACM must be honored. Abstracting with credit is permitted. To copy otherwise, or republish, to post on servers or to redistribute to lists, requires prior specific permission and/or a fee. Request permissions from permissions@acm.org.

HPCAsia2020, January 15-17, 2020, Fukuoka, Japan

(c) 2020 Association for Computing Machinery.

ACM ISBN 978-1-4503-7236-7/20/01 . \$15.00

https://doi.org/10.1145/3368474.3368481

\section{INTRODUCTION}

The algebraic multigrid (AMG) method [4] is an excellent solver and is one of the most effective preconditioners of the conjugate gradient (CG) method for solving systems of linear equations derived from discretized elliptic-type partial differential equations. Among the most well-known preconditioners, namely the weighted Jacobi, symmetric Gauss-Seidel incomplete Cholesky, and AMG, the latter is advantageous in that it has a higher convergence ratio than the other preconditioners on suitable applications.

The advantage of AMG is realized by the combination of smoother and coarse grid correction. The AMG method classifies error components into algebraically non-smooth and smooth errors. The former is eliminated by a smoother. The latter error is eliminated by constructing hierarchically coarse grid spaces, eliminating the non-smooth errors in each grid space, and mapping the algebraically smooth error to the coarser grid space. The high convergence rate of $A M G$ is attained by efficiently removing smooth errors that are difficult to eliminate by using other methods.

An effective smoother is characterized by its efficiency in eliminating non-smooth errors, computational cost, and parallelizability as important features. The ability to efficiently remove the nonsmooth error improves the convergence ratio of the CG method. Moreover, because the execution time of the smoother occupies a non-negligible proportion of the execution time of the entire AMG preconditioned CG (AMG-CG), its low computational cost and parallelizability contribute to the improvement of the overall execution performance.

Although the Gauss-Seidel (GS) method is a fundamental sequential smoother of AMG, and various parallelization efforts have been made based on this method. Multi-color Gauss-Seidel (MCGS) [1][13][17] is a method designed to exploit the parallelism of the GS method, it leads to inferior data locality, which decreases the cache hit rate. The block multi-color Gauss-Seidel (BMC-GS) [2] [10] improves the data locality such that it equals that of sequential GS.

In this paper, we propose the multiplicative Schwartz-type block multi-color Gauss-Seidel (MS-BRB-GS) smoother, which has a higher convergence ratio and data locality than BMC-GS. This smoother functions by repeatedly applying GS sweeps to each block. This approach improves the convergence ratio of AMG-CG in exchange 
for an increased number of floating-point number calculations. Despite the increased amount of computation, the increase in computational time is mitigated by the high cache hit rate of the proposed method.

The design of our proposed MS-BMC-GS smoother includes a novel coloring algorithm and methodology for constructing optimal blocks. The coloring algorithm achieves a coloring that balances the number of blocks of each color while using a minimum number of colors, which maximizes the parallelism and ensures that the computational load is equally distributed among parallel processes. Furthermore, to construct suitable blocks in MS-BMCGS, we apply multiply constrained minimum edge-cutting graph partitioning to improve the convergence ratio of AMG-CG and to equally distribute the computational load.

The contribution of the study presented in this paper is the establishment of a method that achieves a high convergence ratio, data locality, and parallelizability. The evaluation result shows $18 \%$ performance improvement compared with the existing method in the best case.

The remainder of this paper is organized as follows: Section 2 briefly reviews the AMG preconditioner and introduces the requirements of the smoother. Section 3 discusses the limitations of existing GS smoothers. Section 4 describes our method, MS-BMCGS, with the novel coloring algorithm. Section 5 presents the evaluation results. Section 6 introduces related work. Section 7 concludes this paper.

\section{EFFECTIVE SMOOTHER FOR AMG PRECONDITIONER}

In this section, we discuss the prerequisite and requirements for an effective smoother. For the purpose of the discussion, we introduce AMG, which is based on smoothed aggregations [18] and define some conditions.

\subsection{AMG method}

For reference, we briefly introduce the basic idea and present the processes of AMG.

In our study, we consider solving a system of linear equations in $n^{h}$-dimensions as

$$
A^{h} u=b^{h}
$$

where $A^{h}$ denotes the original symmetric coefficient matrix of the target system of linear equations, $\boldsymbol{u}$ denotes the exact solution, and $\boldsymbol{b}^{\boldsymbol{h}}$ denotes the right-hand vector. We also define an element of the coefficient matrix $A^{h}$ as $a_{i j}$. On this equation, an error equation is constructed as

$$
A e^{h}=r^{h}, \because e^{h}=u-x^{h}, r^{h}=b^{h}-A^{h} x^{h} .
$$

Here, $x^{h}$ denotes an approximate solution. Then, we define an error $\tilde{\boldsymbol{e}}^{\boldsymbol{h}}$, which constructs some components that are included in the $\boldsymbol{e}^{\boldsymbol{h}}$ and satisfy the following condition.

$$
M^{h} \tilde{\boldsymbol{e}}^{h} \approx \tilde{\boldsymbol{e}}^{h}
$$

Here, $\boldsymbol{M}^{\boldsymbol{h}}$ denotes an iterative matrix of a basic iterative method such as weight-Jacobi or GS. These iterative methods are known as smoothers in AMG. Equation (3) means that the error is not updated by the smoother. It is difficult to obtain a more optimal

$$
\begin{aligned}
& x^{h} \leftarrow\left(D^{h}\right)^{-1} b^{h}+\left(M^{h}\right)^{\text {pre }} x^{h} \\
& \text { : Pre-smoothing on level } 1 \\
& r^{h} \leftarrow b^{h}-A^{h} x^{h}: \text { Calculating residual vector } \\
& \boldsymbol{r}^{2 \boldsymbol{h}} \leftarrow \boldsymbol{P}_{\boldsymbol{h}}^{2 \boldsymbol{h}} \boldsymbol{r}^{\boldsymbol{h}}: \text { Restriction from level } 1 \text { to } 2 \\
& x^{2 h} \leftarrow\left(D^{2 h}\right)^{-1} r^{2 h}+\left(M^{2 h}\right)^{p r e} x^{2 h} \\
& \text { : Pre-smoothing on level } 2 \\
& b^{L h} \leftarrow P_{(L-1) h}^{L h} r^{(L-1) h} \\
& \boldsymbol{e}^{L \boldsymbol{h}} \leftarrow\left(A^{L h}\right)^{-1} b^{L \boldsymbol{h}}: \text { Solve } \\
& x^{(L-1) h} \leftarrow x^{(L-1) h}+\left(P_{(L-1) h}^{L h}\right)^{t} e^{L h} \\
& \text { : } \\
& x^{2 h} \leftarrow\left(D^{2 h}\right)^{-1} b^{2 h}+\left(M^{2 h}\right)^{p o s t} x^{2 h} \\
& \text { : Post-smoothing on level } 2 \\
& x^{h} \leftarrow x^{h}+\left(P_{h}^{2 h}\right)^{t} e^{2 h} \\
& \text { : Prolongation from level } 2 \text { to } 1 \\
& \boldsymbol{x}^{\boldsymbol{h}} \leftarrow\left(D^{\boldsymbol{h}}\right)^{-1} b^{\boldsymbol{h}}+\left(M^{\boldsymbol{h}}\right)^{\text {post }} \boldsymbol{x}^{\boldsymbol{h}} \\
& \text { : Post-smoothing on level } 1
\end{aligned}
$$

Figure 1: Process of L-level V-cycle AMG

approximate solution by applying the smoother. The error $\tilde{\boldsymbol{e}}^{\boldsymbol{h}}$ is known as an algebraically smooth error.

In the AMG method, we prepare $L$ sparse grid spaces based on the original grid space. We eliminate the algebraically non-smooth error on the finer grid, and map the algebraically smooth error to the coarser grid. This approach enables all errors to be eliminated effectively. Figure 1 shows the process of the V-cycle AMG. Here, $P_{(l-1) h}^{l h}$ denotes a mapping (restriction) operator from the $l-1$ level to the $l$ level, and $\left(M^{l \boldsymbol{h}}\right)^{\text {pre }}$ and $\left(\boldsymbol{M}^{\boldsymbol{l} \boldsymbol{h}}\right)^{\text {post }}$ denote the iterative matrices of the pre- and post-smoothing processes, respectively. The coefficient matrix $A^{l \boldsymbol{h}}$ on the coarser levels are constructed with a Galerkin condition as

$$
A^{l h}=P_{(l-1) h}^{l h} A^{(l-1) h}\left(P_{(l-1) h}^{l h}\right)^{t} .
$$

\subsection{Smoothed aggregation}

A smoothed aggregation is a method to calculate the effective restriction operator. In this section, we introduce the computational process of the smoothed aggregation.

We define a graph $\boldsymbol{F}^{\boldsymbol{h}}\left(\boldsymbol{V}, \boldsymbol{E}, \boldsymbol{E}_{\boldsymbol{w}}\right)$ which represent the coefficient matrix $A^{h}$ of the equation (1). Then, we apply the aggregation to 
the graph $F^{h}$ as follows:

$$
\begin{array}{r}
\boldsymbol{V}\left(\boldsymbol{F}^{\boldsymbol{h}}\right)=\left\{1,2, \cdots, n^{h}\right\} \\
\boldsymbol{E}\left(\boldsymbol{F}^{\boldsymbol{h}}\right)=\left\{i j \mid a_{i j} \neq 0\right\} \\
\boldsymbol{E}_{\boldsymbol{w}}\left(\boldsymbol{F}^{\boldsymbol{h}}\right)=\left\{a_{11}, a_{12}, \cdots, a_{n^{h} n^{h}}\right\},
\end{array}
$$

where $\boldsymbol{V}\left(\boldsymbol{F}^{\boldsymbol{h}}\right), \boldsymbol{E}\left(\boldsymbol{F}^{\boldsymbol{h}}\right)$, and $\boldsymbol{E}_{\boldsymbol{w}}\left(\boldsymbol{F}^{\boldsymbol{h}}\right)$ denote the set of nodes, edges, and weights of the edges in the graph $F^{\boldsymbol{h}}$, respectively, and $i j$ denotes the edge between element $i$ and $j$. The graph $F^{\boldsymbol{h}}$ is derived from the original coefficient matrix $A^{h}$. To construct a coarser grid collection, we separate the graph $\boldsymbol{F}^{\boldsymbol{h}}$ into $n^{2 h}$ parts. This process is known as $n^{2 h}$ graph partitioning and has the following conditions.

$$
\begin{aligned}
V\left(C^{h}\right)= & \left\{c_{1}^{h}, c_{2}^{h}, \cdots, c_{n^{2 h}}^{h}\right\}\left(c_{i}^{h} \neq \emptyset\right) \\
& \cup_{i=1}^{n^{2 h}} c_{i}^{h}=V\left(F^{h}\right) \\
& c_{i}^{h} \cap c_{j}^{h}=\emptyset, \text { if } i \neq j,
\end{aligned}
$$

where $C^{h}(V, E)$ denotes the $n^{2 h}$ partitioned graph and the result of the aggregation.

The restriction operator is expressed as follows.

$$
\begin{array}{r}
\left(\hat{P}_{h}^{2 h}\right)_{i, j}= \begin{cases}1 & \text { if } j \in \boldsymbol{c}_{i}^{\boldsymbol{h}} \\
0 & \text { otherwise }\end{cases} \\
\boldsymbol{P}_{\boldsymbol{h}}^{2 \boldsymbol{h}}=\left(\boldsymbol{M}^{\boldsymbol{h}}\right)^{\boldsymbol{w} J} \hat{\boldsymbol{P}}_{\boldsymbol{h}}^{2 \boldsymbol{h}},
\end{array}
$$

where $\left(M^{h}\right)^{w J}$ denotes the iterative matrix of the weighted Jacobi. We expect this calculation to enable the operator $P_{h}^{2 h}$ to restrict the smooth error from the original grids to the coarse grids.

\subsection{Prerequisite of the smoother}

Using the V-cycle AMG method as the preconditioner of the CG method requires the iterative matrix of AMG to be symmetric. In this section, we verify the symmetry of the AMG method and a prerequisite of the smoother.

In the section2.1, we introduce the process of the V-cycle AMG. A single iterative update is expressed as follows.

$$
\begin{aligned}
x^{h} \leftarrow & \left(D^{h}\right)^{-1} b^{h}+\left(M^{h}\right)^{p o s t}\left[\left(D^{h}\right)^{-1} b^{h}\right. \\
& +\left(M^{h}\right)^{p r e} x^{h}+\left(P_{h}^{2 h}\right)^{t}\left(A^{2 h}\right)^{-1} P_{h}^{2 h} \\
& \left.\left\{b^{h}-A^{h}\left(\left(D^{h}\right)^{-1} b^{h}+\left(M^{h}\right)^{p r e} x^{h}\right)\right\}\right]
\end{aligned}
$$

The matrix $A^{2 h}$ can be replaced by the same updating process, recursively. The iterative matrix of AMG is calculated as follows.

$$
\begin{aligned}
M^{A M G}=\left(M^{h}\right)^{\text {post }}\{I+ & \\
& \left.\left(P_{h}^{2 h}\right)^{t}\left(A^{2 h}\right)^{-1} P_{h}^{2 h} A^{h}\right\}\left(M^{h}\right)^{\text {pre }}
\end{aligned}
$$

The matrix $I$ denotes the identity matrix. Then, we assume the coefficient matrix on the coarse grid $A^{2 h}$ to be symmetric. To retain the symmetry, pre- and post-smoothing must satisfy the following condition.

$$
\left(M^{h}\right)^{\text {post }}=\left\{\left(M^{h}\right)^{\text {pre }}\right\}^{t}
$$

The coefficient matrix on the coarse grid $A^{2 h}$ is calculated as equation (4). The symmetricity of $A^{2 h}$ is also retained by applying the same condition (13) on the coarser grids.

\subsection{Requirements of the smoother}

The requirements of the smoother are as follows.

- Parallelizable

- Shorter computational time

- Ability to effectively eliminate non-smooth errors

The iterative processes of the AMG method, excluding the smoother, are all parallelizable. Therefore, the extent to which the AMG can be paralellized depends on the smoother. In addition, the contribution of the smoother to the computational cost of AMG is the largest. In some experiments, the cost of the smoother is approximately $30 \%$ of that of the entire AMG-CG method. Therefore, selecting a faster smoother would reduce the computational time of AMG. The capacity of the smoother to eliminate algebraically non-smooth errors is also important. Improving the performance in terms of eliminating algebraically non-smooth errors would reduce the number of iterations of AMG-CG.

The weighted Jacobi and GS are well-known smoothers. However, whereas the weighted Jacobi is easy to parallelize, this is known to be difficult to achieve for the GS smoother. The computational cost of the weighted Jacobi and GS is similar. In terms of their ability to eliminate an algebraically non-smooth error, the GS smoother is more efficient than the weighted Jacobi. Previous studies have shown that the convergence ratio of AMG-CG with the GS smoother is more than twice as high as that of the weighted Jacobi smoother [5]. This motivated our decision to choose the GS smoother in this study.

\section{EXISTING PARALLEL GS SMOOTHERS}

In this section, we introduce existing parallelized GS smoothers, which form the basis of MS-BMC-GS.

\subsection{Multi-color GS}

Multi-coloring parallelization is a practical method for parallelizing the GS smoother. Figure 2(a) shows an example graph of the set of nodes defined by equation (5), and figure 2(b) shows an example of a multi-colored graph. The updated result $\dot{x}_{i}^{h}$ of the GS smoother is expressed as

$$
\dot{x}_{i}^{h}=\frac{1}{a_{i, i}^{h}}\left(b_{i}^{h}-\sum_{j=1}^{i-1} a_{i, j}^{h} \dot{x}_{j}^{h}-\sum_{j=i+1}^{n^{h}} a_{i, j}^{h} x_{j}^{h}\right) .
$$

Here, element $x_{i}$ is updated by using updated elements $x_{1}, x_{2}, \cdots, x_{i-2}, x_{i-1}$. It is not possible to parallelize the GS smoother in the given order because of data dependencies. If we apply multi-coloring to the graph $F^{h}$ by using any algorithm, each node would be assigned an arbitrary color. This approach would ensure that the color of a node differs from that of the other nodes that have relationships with this node. Thus, the application of multi-coloring prevents 
the formation of relationships between nodes that are assigned the same color. This condition allows us to parallelize the process of updating nodes colored with the same color.

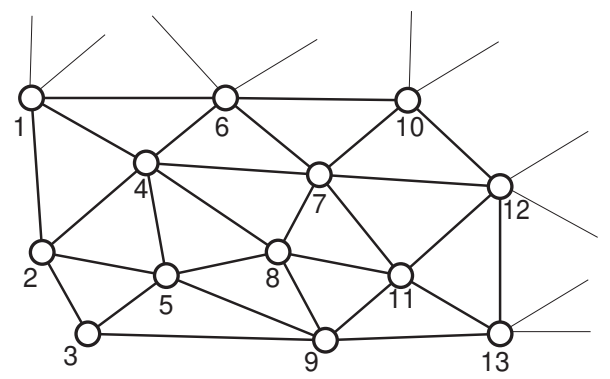

(a) original target

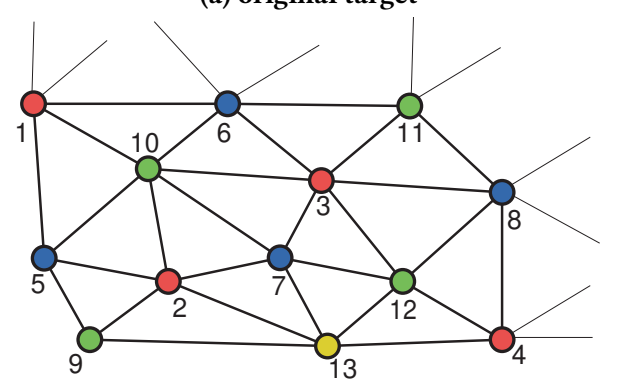

(b) Multi-coloring

Figure 2: Example of the multi-coloring

\subsection{Block Multi-color GS}

The block multi-color Gauss-Seidel (BMC-GS) smoother is a modified version of the multi-colored Gauss-Seidel (MC-GS) smoother. BMC-GS enhances the convergence ratio of AMG-CG and the data locality compared with MC-GS.

To apply block multi-coloring, we separate all nodes into some blocks (figure 4(a)). After that, we apply the multi-coloring to all the blocks (figure 4(b)). Generally, the sequential GS smoother converges faster than MC-GS. Employing a larger block size would achieve a convergence ratio closer to that with the sequential GS smoother because of similar data dependencies. On the other hand, the use of a smaller block size would obtain a convergence ratio closer to that with MC-GS. As a result, it becomes possible to increase the convergence ratio with the BMC-GS such that it is higher than that of MC-GS. The data locality is also increased by a blocking technique [15]. Figure 3 shows a sample implementation of BMC-GS.

\section{MULTIPLICATIVE SCHWARTZ-TYPE BMC-GS}

In this section, we propose the multiplicative type block multicoloring Gauss-Seidel(MS-BMC-GS) smoother. The advantages of MS-BMC-GS are an improved convergence ratio and data locality compared with BMC-GS. We explain why MS-BMC-GS offers these advantages, verify the symmetricity of MS-BMC-GS, and introduce optimizations.
Do $j=1$, num_colors

!\$OMP parallel do

Do $i=1$, num_blocks $(j)$

Updating elements in the $i$ th block Enddo

Enddo

Figure 3: Example of the implementation of BMC-GS

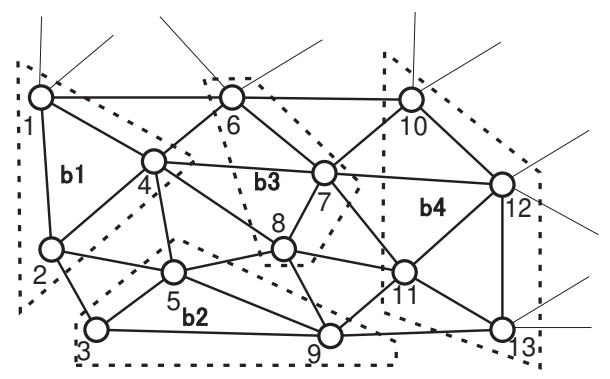

(a) Separating the target graph into four blocks

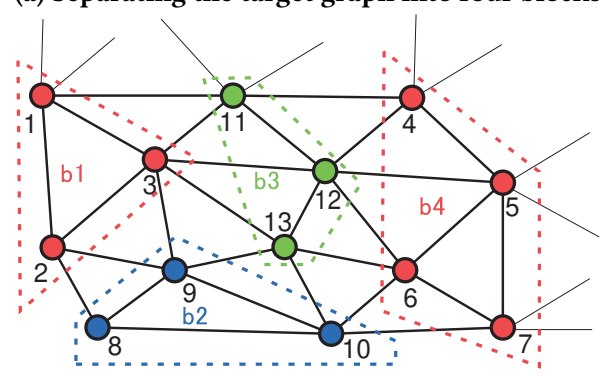

(b) Block multi-coloring

Figure 4: Example of block multi-coloring

\subsection{Basic idea}

The principle that forms the basis of MS-BMC-GS is that the block size is purposefully specified to be smaller than the cache size of the CPU and to carry out GS sweeps on each block. This approach achieves further improvement of the convergence ratio and datalocality compared with the BMC-GS.

With respect to the multigrid method, increasing the number of smoothing steps improves the convergence ratio of AMG-CG. Simultaneously, this approach also increases the computational time per iterative step. As mentioned in section 2.4, we define the computational time of the smoother as $30 \%$ of the overall computational time of AMG-CG. Then, the computational time per iteration with $\beta$ time BMC-GS smoothing (denoted as $\mathrm{BMC}-\mathrm{GS}(\beta)$ ) is expressed as

$$
t^{B}(\beta)=(\underbrace{0.3 \beta}_{\text {Smoother }}+\underbrace{0.7}_{\text {The other part }}) \times t^{B}(1) .
$$

To reduce the computational time by increasing $\beta$, the number of iterations of the AMG preconditioned CG must be $10 /(3 \beta+7)$ times 
less. That is, if we use BMC-GS(2), the number of iterations must be 0.77 times less than those required by BMC-GS(1).

MS-BMC-GS, which we propose in this paper, is a modified version of BMC-GS. We show the difference between BMC-GS and MS-BMC-GS in figure 5. The difference in the implementation between BMC-GS and MS-BMC-GS is shown in figure 6. In the block multi-coloring process, we determine the number of blocks such that the required memory resources for each block are smaller than the cache size in the CPU. Subsequently, we apply $\alpha$ times GS sweeps to each block, repeatedly. Hereinafter, we denote MSBMC-GS with $\alpha$ times sweeps on each block as MS-BMC-GS $(\alpha)$.

Generally, if $\beta=\alpha$, the convergence ratio of $\operatorname{BMC}-\mathrm{GS}(\beta)$ is higher than that of MS-BMC-GS $(\alpha)$. However, the execution time required for the second and later sweeps on each block is much less than that required for the first sweep because of on-cache computations. Here, we assume that the execution time of the second and later sweeps is $\delta$ times faster than the first sweep. The computational time of MS-BMC-GS $(\alpha)$ is expressed as

$$
t^{M}(\alpha)=\{\underbrace{0.3}_{\text {First GS sweep }}+\underbrace{\delta * 0.3 *(\alpha-1)}_{\text {Later GS sweeps }}+0.7\} \times t^{M}(1)
$$

To achieve a reduction in the computational time would require MS-BMC-GS to use fewer than $10 /\{3+3 \delta(\alpha-1)+7\}$ iterations. For example, if the value of $\delta$ was 0.25 for an arbitrary system, the required number of iterations for improving the performance with MS-BMC-GS(2) would be 0.93 times less than BMC-GS(1). In comparison, it would be easier to improve the performance of MS$\operatorname{BMC}-\mathrm{GS}(\alpha)$ than that of BMC-GS $(\alpha)$.

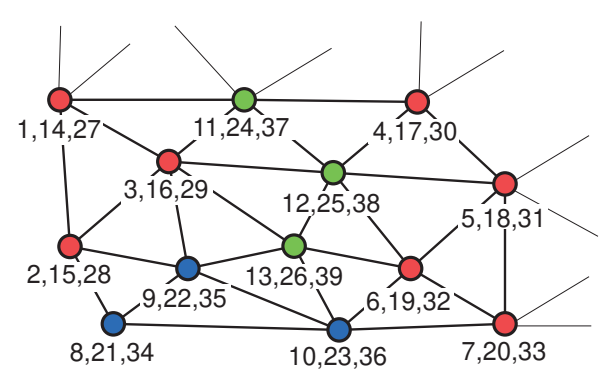

(a) BMC-GS(3)

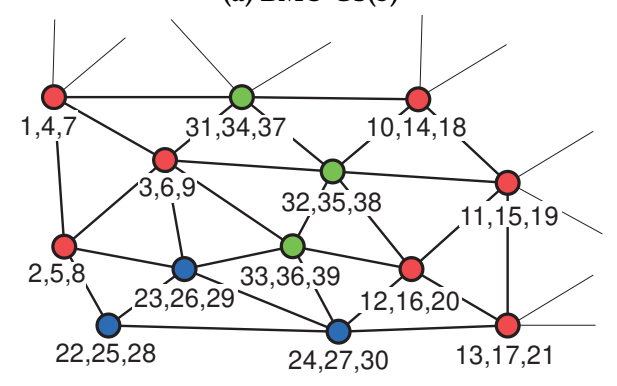

(b) MS-BMC-GS(3)

Figure 5: Differential between BMC-GS(3) and MS-BMCGS(3)
Do $j=1$, num_colors

!\$OMP parallel do

Do $i=1$, num_blocks $(j)$

Do rep_gs $=1, \alpha$

Updating elements in the $i$ th block

Enddo

Enddo

Enddo

Figure 6: Example of the implementation of MS-BMC-GS (the difference between MS-BMC-GS and BMC-GS is shown in red)

\subsection{Symmetry of AMG preconditioner with MS-BMC-GS}

In this section, we present the condition for retaining the symmetry of the AMG preconditioner with MS-BMC-GS. Retention of the symmetry requires us to apply the same number of GS sweeps to the pre- and post-MS-BMC-GS smoother.

We denote the reordered matrix from $A^{h}$ based on the result of using $c$ colors block multi-coloring as

$$
\begin{aligned}
\bar{A}^{\boldsymbol{h}} & =\bar{D}^{\boldsymbol{h}}-\bar{U}^{\boldsymbol{h}}-\left(\bar{U}^{\boldsymbol{h}}\right)^{t} . \\
& =\left(\begin{array}{cccc}
\bar{D}_{1,1}^{\boldsymbol{h}} & \bar{U}_{1,2}^{\boldsymbol{h}} & \cdots & \bar{U}_{1, c}^{\boldsymbol{h}} \\
\left(\bar{U}_{1,2}^{\boldsymbol{h}}\right)^{t} & \bar{D}_{2,2}^{\boldsymbol{h}} & \cdots & \bar{U}_{2 m}^{\boldsymbol{h}} \\
\vdots & \vdots & \ddots & \vdots \\
\left(\bar{U}_{1, c}^{\boldsymbol{h}}\right)^{t} & \left(\bar{U}_{2, c}^{\boldsymbol{h}}\right)^{t} & \cdots & \bar{D}_{c, c}^{\boldsymbol{h}}
\end{array}\right) .
\end{aligned}
$$

Figure 7 shows an example of the $\bar{A}^{h}$. Then, we denote the iterative matrix of the sequential GS based on the $\overline{\boldsymbol{D}}^{\boldsymbol{h}}$ as $\left(\overline{\boldsymbol{M}}^{\boldsymbol{h}}\right)^{G S}$. The iterative matrix of MS-BMC-GS is expressed as

$$
\left(\boldsymbol{M}^{\boldsymbol{h}}\right)^{M B M}=\left[\left\{\left(\overline{\boldsymbol{M}}^{\boldsymbol{h}}\right)^{G S}\right\}^{\alpha}-\left(\overline{\boldsymbol{U}}^{\boldsymbol{h}}\right)^{t}\right]^{-1} \overline{\boldsymbol{U}}^{\boldsymbol{h}}
$$

Hence, the backward MS-BMC-GS $(\alpha)$ is expressed as follows.

$$
\left(\boldsymbol{M}^{\boldsymbol{h}}\right)^{r M B M}=\left[\left\{\left(\bar{M}^{\boldsymbol{h}}\right)^{r G S}\right\}^{\alpha}-\overline{\boldsymbol{U}}^{\boldsymbol{h}}\right]^{-1}\left(\overline{\boldsymbol{U}}^{\boldsymbol{h}}\right)^{t}
$$

In the process of the backward MS-BMC-GS $(\alpha)$, we apply the backward GS to each block and update all blocks in the reverse order in which they were initially colored. Then, we perform forward MS-BMC-GS as pre-smoother and backward MS-BMC-GS as postsmoother. As we mentioned in section 2.3, the prerequisite of the smoother is that post-smoothing must be the transposed process of pre-smoothing. To ensure compliance with the condition

$$
\left(\boldsymbol{M}^{\boldsymbol{h}}\right)^{M B M}=\left\{\left(\boldsymbol{M}^{\boldsymbol{h}}\right)^{r M B M}\right\}^{t}
$$


the same number of GS sweeps need to be applied for pre- and post-MS-BMC-GS smoothing.
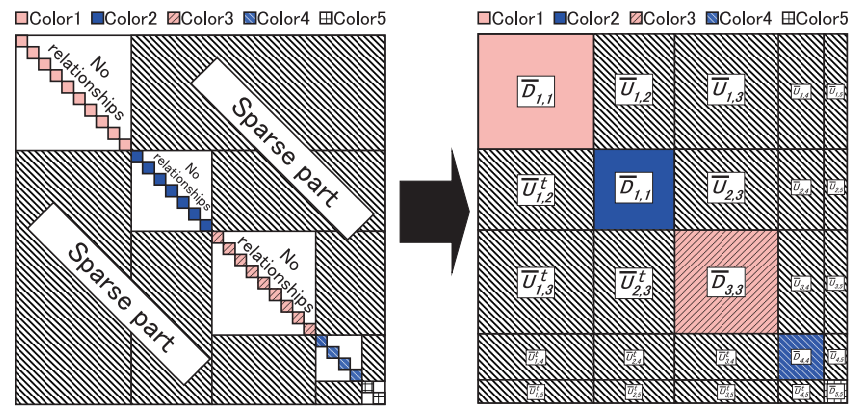

Figure 7: Reordered and separated coefficient matrix based on the result of using five colors for block multi-coloring

\subsection{Coloring algorithm for MS-BMC-GS}

In this section, we introduce the coloring algorithm we implement. The algorithm is selected to obtain the desired degree of parallelism and load balance on each color to ensure the effectiveness of MS-BMC-GS. This algorithm combines a greedy approach with algebraic block multi-coloring[10].

Two parameters need to be specified for block multi-coloring: the size of the block and the number of colors. Generally, a larger block and more colors improve the convergence ratio of AMG-CG, whereas a smaller block and fewer colors increase the degree of parallelism. MS-BMC-GS requires the block size to be smaller than the cache size. Under this condition, the block size is selected as the largest that would allow a higher convergence ratio of AMGCG. The size of the block is determined by the size of the cache. Therefore, we ensure the degree of parallelism by the number of colors.

We denote a target graph $G(V, E)$ for coloring. The graph $G$ is the $n^{b}$ partitioned graph based on $\boldsymbol{F}^{\boldsymbol{h}}$ of equation (5). For MS-BMCGS, we propose the aforementioned modified algorithm, which combines a greedy approach with algebraic multi-coloring. Figure 8 shows the pseudo-code of the implemented coloring algorithm. In this figure, $\boldsymbol{R}$ (icolor) denotes the set of nodes colored with icolor. The greedy algorithm uses fewer colors to color all blocks. However, the number of blocks of each color differs considerably. Generally, small color ID (icolor in the figure 8) is assigned to many blocks and large color ID is assigned to few blocks. In many situations, the number of blocks colored with the largest color ID is less than the degree of parallelism. The algebraic multi-coloring algorithm produces the same number of blocks in each color. Thus, we apply algebraic multi-coloring and then apply the idea of the greedy algorithm to control the number of colors.

\subsection{Optimal blocks}

In this section, we discuss a methodology that allows for the construction of optimal blocks for MS-BMC-GS.

The block construction needs to meet the following requirements with the aim of increasing the convergence ratio of AMGCG and the performance:

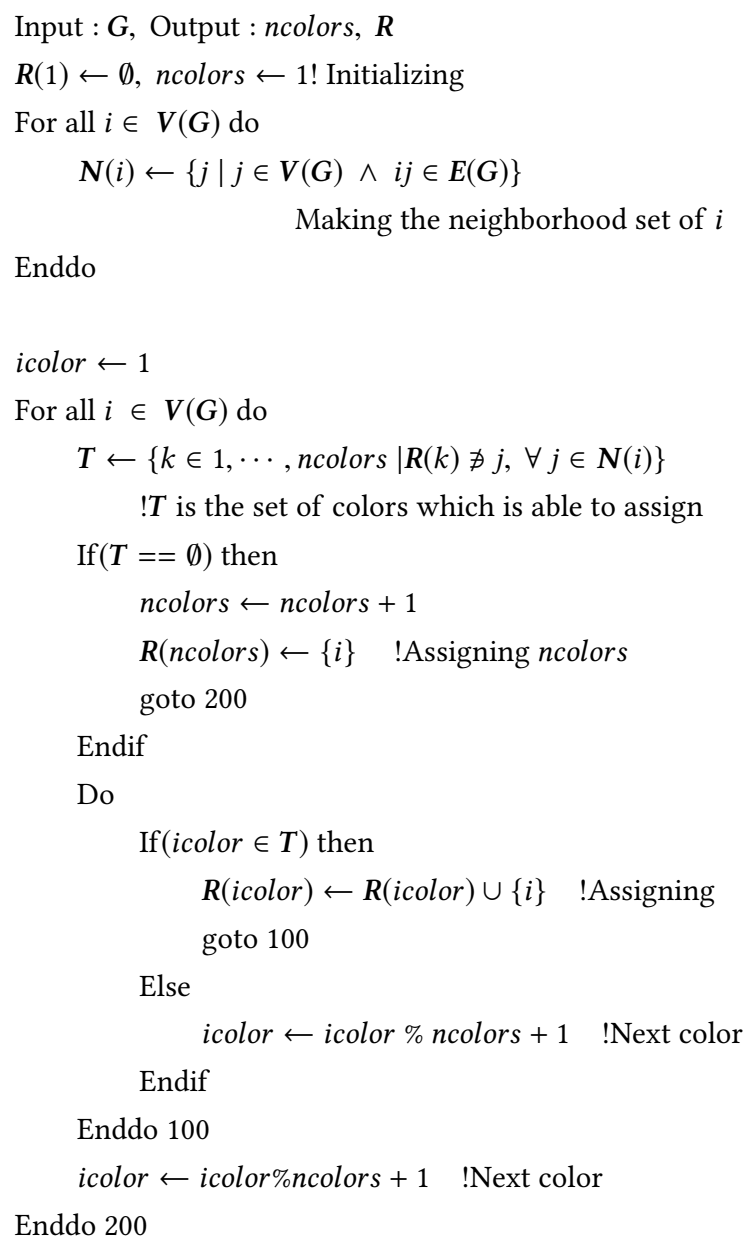

Figure 8: Multi-coloring algorithm implemented in this work

\section{Optimize the block size}

As mentioned in 4.1, the memory required for each block must be less than the cache size. However, because of the need to repeatedly use a GS sweep in each block, a larger block promotes convergence.

Maximize the couplings in each block

The number of relationships within the blocks is maximized to maximize the effect of the GS sweeps.

Balance the computational cost among blocks

An increase in the number of GS sweeps increases the load imbalance among the blocks. Balanced blocks are important for higher performance.

We separate all the nodes into $n^{b}$ blocks by using any graph partitioning algorithm based on the aggregation result. When applying the blocking technique, we have the option of either constructing the blocks by ignoring or by considering the aggregation result. Previously [3] it was shown that blocks that depend on the 
aggregation result have improved convergence. Therefore, we decided to apply the blocking technique accordingly. Then, we define the aggregation result as $C^{\boldsymbol{h}}$ and redefine the graph $G$ as the $n^{b}$ partitioned graph based on the $C^{h}$. We implemented an existing aggregation process [18].

The easiest method for constructing blocks is to select the set $\boldsymbol{c}_{i}^{\boldsymbol{h}}$ in lexicographical order for addition to a block. However, this method makes it difficult to consider the requirements. Thus, we focused on using the METIS [11] library, which produces graph partitioning with minimized edge-cutting. Maximizing the relationships within blocks and minimizing the relationships among blocks has the same meaning. METIS also allows partitioning according to multiple constraints. If we use the weights of the nodes as a constraint, METIS attempts to balance the weights of the partitioned sets. In this regard, we considered the computational cost of each aggregated subset as the weight of the node to enable the computational cost of the partitioned parts to be balanced.

We calculate the number of blocks as

$$
n_{b}=n^{2 h} \times \frac{\gamma}{\rho \times s_{c}}
$$

where $\gamma$ denotes the average memory consumption for updating all the nodes in each aggregated set $c_{i}^{h}, s_{c}$ denotes the size of the cache memory of a system, and $\rho$ denotes an arbitrary factor with the condition $0<\rho<1$. We use the factor $\rho$ to control the memory consumption of each block such that it remains less than the cache size. Then, we expand the graph $C^{\boldsymbol{h}}(\boldsymbol{V}, \boldsymbol{E})$ denoted as the result of aggregation to a weighted graph $C^{h}\left(\boldsymbol{V}, \boldsymbol{E}, \boldsymbol{V}_{\boldsymbol{w}}, \boldsymbol{E}_{\boldsymbol{w}}\right)$. The value of $V_{w}\left(C^{h}\right)$ is calculated as

$$
V_{w}\left(C^{h}\right)_{i}=\left|E\left(c_{i}^{h}\right)\right|
$$

Here, $V_{w}\left(C^{h}\right)_{i}$ is expressed as the number of edges including the set $\boldsymbol{c}_{i}^{\boldsymbol{h}}$. Hereinafter, we denote partitioning in lexicographical order as LEX, unconstrained METIS partitioning as METIS_P, and constrained METIS partitioning with node weights as BALANCE.

We consider the weight of the edges for the construction of the graph partitioning to achieve highly optimized block construction. As mentioned in 2.1, on each level, the smoother is used to eliminate the non-smooth errors. Effective repeated GS sweeps on each block requires each block to preferably contain stronger couplings. Thus, we use the nonzero elements among the aggregated subset as the weights of the edges. We have two options for defining the nonzero elements among subsets. If we focus on the level $l$, we use the nonzero elements of $A^{\boldsymbol{l h}}$ or $A^{(\boldsymbol{l + 1}) \boldsymbol{h}}$ for the weights. Then we denote the weights of the edges based on $A^{\boldsymbol{l h}}$ and $A^{(\boldsymbol{l}+1) \boldsymbol{h}}$ as $E 1_{w}\left(C^{h}\right)$ and $E 2_{w}\left(C^{h}\right)$, respectively. We calculate the $E 1_{w}\left(C^{h}\right)$ as

$$
E 1_{w}\left(C^{h}\right)(i j)=\sum E_{w}\left(F^{h}\right)\left(c_{i}^{h}, c_{j}^{h}\right)
$$

We also define $\boldsymbol{E} \mathbf{1}_{\boldsymbol{w}}\left(C^{\boldsymbol{h}}\right)$ as

$$
E 2_{w}\left(C^{h}\right)(i j)=a_{i j}^{2 h}
$$

To use the nonzero elements as constraints for METIS, we have to regularize elements with positive integers. We calculate the regularized weight of the edge $\boldsymbol{E}_{\boldsymbol{w}}^{\prime}\left(C^{\boldsymbol{h}}\right)$ as follows.

$$
\begin{aligned}
w_{\text {min }}=\min & \left\{\boldsymbol{E}_{\boldsymbol{w}}\left(\boldsymbol{C}^{\boldsymbol{h}}\right)\right\} \\
w_{\text {max }}=\max & \left\{\boldsymbol{E}_{\boldsymbol{w}}\left(\boldsymbol{C}^{\boldsymbol{h}}\right)\right\} \\
\boldsymbol{E}_{\boldsymbol{w}}^{\prime}\left(\boldsymbol{C}^{\boldsymbol{h}}\right)(i j)= & \left\{\boldsymbol{E}_{\boldsymbol{w}}\left(\boldsymbol{C}^{\boldsymbol{h}}\right)(i j)-w_{\min }\right\} \\
\times & \left(w_{\text {max }}-w_{\text {min }}\right) \times\left(\omega_{\text {max }}-1.0\right)+1.0,
\end{aligned}
$$

where $\omega_{\max }$ denotes the maximum value of the regularizations. Hereinafter, we denote the weighted edge constraints with $E \mathbf{1}_{w}\left(C^{h}\right)$ as WEIGHT1, and with matrix $E 2_{w}\left(C^{h}\right)$ as WEIGHT2

\section{NUMERICAL EVALUATIONS}

This section presents our evaluation of the effect of MS-BMC-GS on selected systems. We also determine the best methodology for constructing the optimal blocks.

\subsection{Environment}

For the numerical evaluations, we prepared the problems thermal2, parabolic fem, G3 circuit, and Poisson. The thermal2, parabolic fem, and G3 circuit problems are published in the Florida suitesparse matrix collection [6]. The Poisson problem is an original model derived from the 3D finite difference method on a structure grid. The diffusion factors in the Poisson problem are random numbers in the range from 0 to 10,000 . The other properties of the target problems are provided in table 1.

In the AMG method, we used the CRS format for storing the matrices. In the problems, the number of levels of the coarse grid correction is five. The parameter $\varepsilon$ for the aggregation is defined as

$$
\varepsilon=0.01 \times\left(\frac{1}{5}\right)^{(l-1)} .
$$

The weight of the weighted Jacobi for calculating the restriction operator is $2 / 3$. The parameter $\omega_{\max }$ for regularizing the weight of the edge is 100 .

Table 2 shows the specifications of the systems. On any system, we set the parameter $s_{l}$ for controlling the size of the block as $512 \mathrm{~KB}$. The parameter $\rho$ for controlling the margin is set to 0.8 . The number of threads for parallel computation are 16, 80, and 64 threads on the NASU system, Grand Chariot, and Oakforest-PACS supercomputers, respectively.

\subsection{Results}

This section presents the best methodology of the blocking construction for MS-BMC-GS on the NASU system, after which we also show the effect of MS-BMC-GS on each system.

\subsection{Comparison among blocking methodology}

To determine the best blocking methodology for MS-BMC-GS, we computed the balance of the number of floating operations on each block, the convergence ratio, and computational time with LEX, METIS_P, BALANCE, WEIGHT1, 
Table 1: Specifications of target problems

\begin{tabular}{|c|r|r|r|r|}
\hline & Thermal2 & Parabolic FEM & G3 Circuit & Poisson \\
\hline Degree of freedom & $1,228,045$ & 525,825 & $1,585,478$ & $12,499,999$ \\
\hline Number of nonzero & $8,580,313$ & $3,674,625$ & $7,660,826$ & $87,174,987$ \\
\hline
\end{tabular}

Table 2: Specifications of systems

\begin{tabular}{|r|r|r|r|r|}
\hline & System name & NASU & Grand Chariot & Oakforest-PACS \\
\hline \multirow{4}{*}{ Processor } & Model & Xeon Silver & Xeon Gold & Xeon Phi \\
\cline { 2 - 5 } & Numbering & 4110 (Skylake) & 6148 (Skylake) & 7250 (Knights Landing) \\
\cline { 2 - 5 } & Number of cores & 8 (1 socket) & 40 (2 sockets) & 64 (1 socket) \\
\cline { 2 - 5 } & Clock frequency & $2.1 \mathrm{GHz}$ & $2.4 \mathrm{GHz}$ & $1.4 \mathrm{GHz}$ \\
\cline { 2 - 5 } & L1D-cache size & $32 \mathrm{KByte} /$ core & $32 \mathrm{KByte} / \mathrm{core}$ & $32 \mathrm{KByte} /$ core \\
\cline { 2 - 5 } & L2-cache size & $1024 \mathrm{KByte} /$ core & $1024 \mathrm{KByte} /$ core & $1024 \mathrm{KByte} / 2$ cores \\
\cline { 2 - 5 } Memory & L3-cache size & $11,264 \mathrm{KByte} /$ socket & $22,528 \mathrm{KByte} /$ socket & none \\
\cline { 2 - 5 } & Technology & DDR4-2666 & DDR4-2666 & MCDRAM \\
\hline \multirow{2}{*}{ Software } & Size & $48 \mathrm{GByte}$ & $384 \mathrm{GBy} \mathrm{G}$ & $16 \mathrm{GByte}$ \\
\cline { 2 - 5 } & Compiler & Intel FORTRAN & Intel FORTRAN & Intel FORTRAN \\
\cline { 2 - 5 } & Version & 19.0 .1 .144 & 18.0 .3 .222 & 18.0 .1 .163 \\
\hline
\end{tabular}

WEIGHT2 constraints partitioning. We also performed the computations with the combined constraints BALANCE+ WEIGHT1 and BALANCE+WEIGHT2. For the numerical evaluations, we focus on the NASU system. Figure 9 shows the minimum, average, and maximum number of floating operations with each method. Figure 10 shows the number of iterations and the computational time.

As shown in figure 9, the load imbalance with LEX is worse than the method with METIS. In the worst case, with LEX, the difference between the minimum and maximum load imbalance is 3.36 times on the parabolic FEM. By using the balance constraint, the load imbalance is reduced. The differences among BALANCE, BALANCE+WEIGHT1, and BALANCE+WEIGHT2 are small. In the worst case, the difference between the minimum and maximum load imbalance is only 1.11 times with BALANCE on the G3 circuit.

As shown in figure 10, AMG-CG with MS-BMC-GS realizes a higher convergence ratio than that with MS-BMC. The WEIGHT2 constraint achieves faster convergence than any other method with the G3 circuit. In the best case, MS-BMC-GS(3) required 1.53 times less iteration on the G3 circuit. Because of the improvement in the convergence, MS-BMC-GS is faster than BMC-GS on any of the problems. The best blocking methodology for each of the problems is different, with BALANCE, BALANCE+WEIGHT1, or BALANCE+WEIGHT2 being the fastest. The best result was achieved for MS-BMC-GS with BALANCE+WEIGHT1 as the constraint and was $18.0 \%$ faster than BMC-GS with the BALANCE constraint on parabolic FEM.

\subsection{Comparison of the computer systems}

In this section, we consider the effect of MS-BMC-GS on the computational result. Figure 11 shows the computational time required to solve the Poisson problem on each system. For the comparison, we chose BMC-GS(LEX), BMC-GS(BALANCE), and MS-BMC-GS

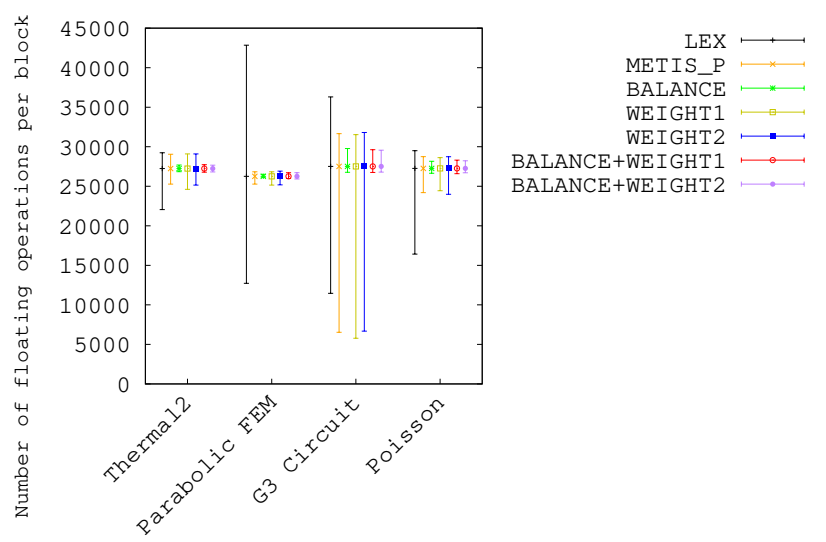

Figure 9: Comparison of load imbalance among blocking methods

with the best $\alpha$ times GS sweeps and blocking methodology. On the NASU system and Grand Chariot system, MS-BMC-GS outperforms BMC-GS. Specifically, MS-BMC-GS performs the computation $4.0 \%$ and $10.9 \%$ faster than BMC-GS(BALANCE) on NASU and Grand Chariot, respectively. On the other hand, on OakforestPACS, MS-BMC-GS is slower than BMC-GS. We noticed that the second and later GS sweeps on each step are not sufficiently faster than the first step on Oakforest-PACS. On Grand Chariot, the second and later GS sweeps are 4.6 times faster than the first GS sweep, whereas on Oakforest-PACS, the second and later GS sweeps are only 1.2 times faster than the first GS sweep. The higher memory and lower L2 cache bandwidth on Oakforest-PACS is considered one of the reasons. 


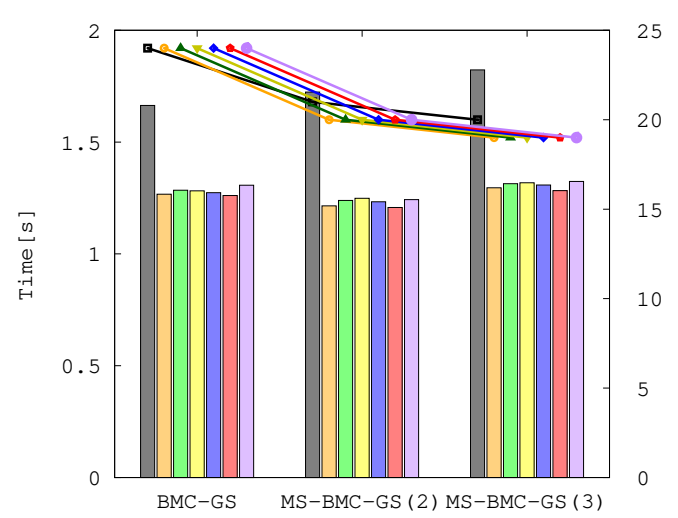

(a) Thermal2

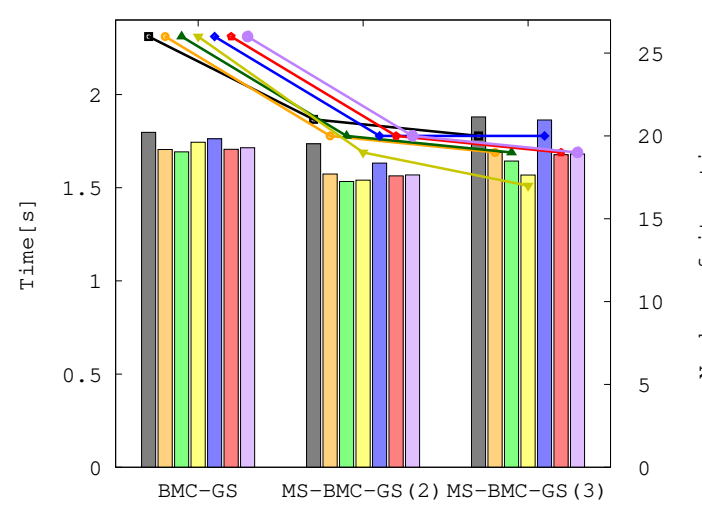

(c) G3 Circuit
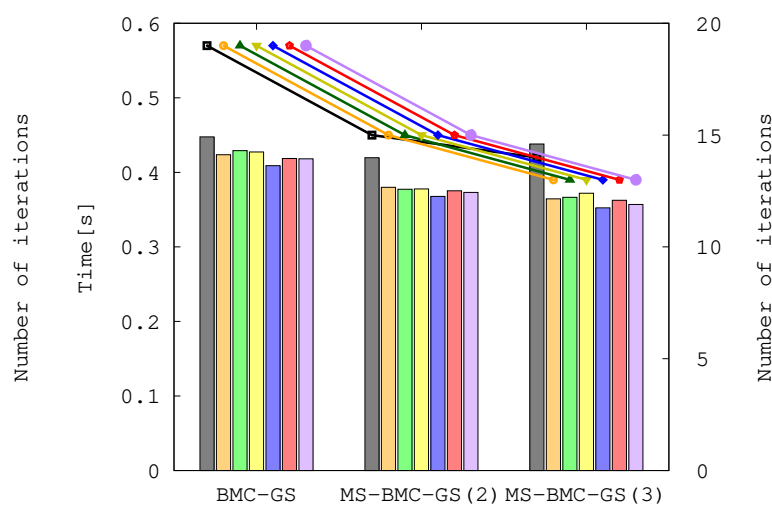

(b) Parabolic FEM

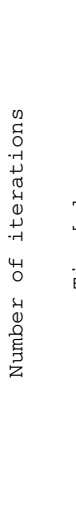

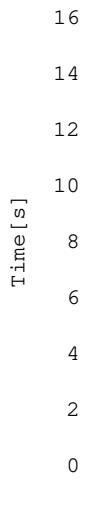

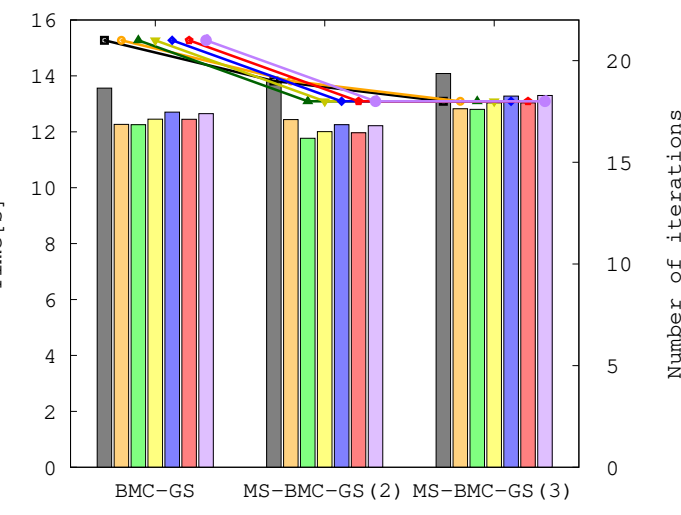

(d) Poisson

Figure 10: Computational time and number of iterations with each blocking method and problem (The lines indicate the number of iterative steps, and the bars the computational time.)

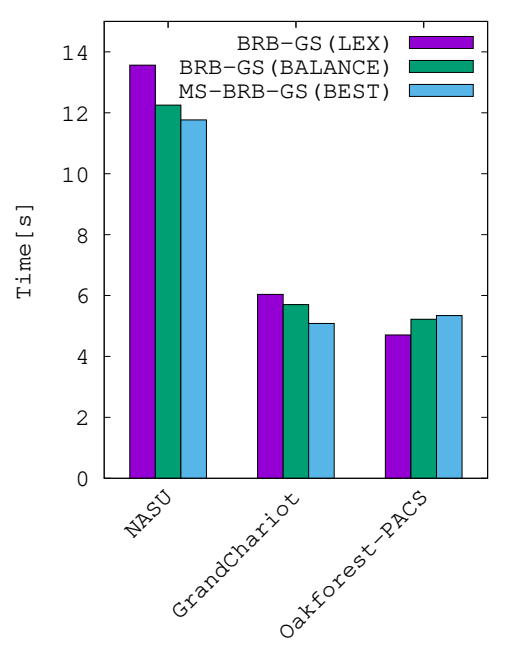

Figure 11: Comparison on each system

\section{RELATED WORK}

Various parallelized GS smoothers, such as the hybrid method [9, 19], MC-GS [1, 7, 13, 17], and BMC-GS [2, 10] have been developed. The hybrid smoother uses the weighted Jacobi to remove the data dependencies of the GS smoother among the separated units. Then, the convergence ratio of AMG-CG with the hybrid smoother approaches that of the weight-Jacobi smoother. In previous studies of MC-GS and BMC-GS, various coloring algorithms and methodologies for constructing blocks were used to realize a convergence ratio similar to that of the sequential GS smoother. Previous studies typically aimed to obtain a convergence ratio similar to that of the sequential GS smoother. With our proposed solution, we realized a higher convergence ratio than that of sequential GS[12].

Cache tiling is an existing approach for improving the cache hit ratio $[8,14,16]$. A target application with the tiling approach produces the same result as the non-tiling approach. An implementation capable of supporting this condition would become more complicated than the original one. The implementation of MS-BMC-GS is simple, as shown in figure 6. The addition of a single loop to BMC-GS enabled us to obtain a result with MS-BMC-GS $(\alpha)$ that differs from that of BMC-GS $(\alpha)$. This difference is permissible because of the implicit method. As a result, MS-BMC-GS is more 
practical than the cache tiling approach as a smoother for the AMG method.

\section{CONCLUSION}

In this paper, we propose the multiplicative Schwartz-type block multi-color GS smoother for the AMG method. This approach improves convergence and data locality. We also discuss a novel coloring algorithm and methodology for constructing optimal blocks for MS-BMC-GS. Based on the numerical evaluations, METIS partitioning with weighted node and edge constraints is suitable for MS-BMC-GS. With the best blocking methodology, MS-BMC-GS showed a performance improvement of $18.0 \%$ compared with BMCGS on the NASU system.

By considering the methodology for constructing the optimal blocks, we found that the constraints for the partitioning improve the convergence ratio or load imbalance. The result implies that a methodology that achieves both a high convergence ratio and load imbalance has the possibility of further improving the performance of AMG-CG. We aim to research the best methodology in future work.

On the other hand, on Oakforest-PACS, MS-BMC-GS is slower than BMC-GS. This is attributed to the slower acceleration of the second and later GS Sweeps because of SIMDization being disabled. In future work, we plan to change the storage format for the sparse matrices to improve the performance of the second and later GS sweeps.

\section{REFERENCES}

[1] Loyce Adams and J Ortega. 1982. A multi-color SOR method for parallel computation. In ICPP. Citeseer, 53-56.

[2] Mark Adams, Marian Brezina, Jonathan Hu, and Ray Tuminaro. 2003. Parallel multigrid smoothing: polynomial versus Gauss-Seidel. f. Comput. Phys. 188, 2 (2003), 593-610

[3] Matthias Bolten and Hannah Rittich. 2018. Fourier analysis of periodic stencils in multigrid methods. SIAM fournal on Scientific Computing 40, 3 (2018), A1642A1668.

[4] Achi Brandt, Steve F McCormick, and John Huge. 1985. Algebraic multigrid (amg) for sparse matrix equations. Sparsity and its Applications (1985), 257-284.

[5] William L Briggs, Van Emden Henson, and Steve F McCormick. 2000. A Multigrid Tutorial Second Edition. SIAM, Philadelphia, PA.

[6] Timothy A. Davis and Yifan Hu. 2011. The university of Florida sparse matrix collection. ACM Trans. Math. Softw. 38, 1 (2011), 1.

[7] Shun Doi and Takumi Washio. 1999. Using multicolor ordering with many colors to strike a better balance between parallelism and convergence. In Proceedings of RIKEN Symposium on Linear Algebra and its Applications. 19-26.

[8] Takeshi Fukaya and Takeshi Iwashita. 2018. Time-space tiling with tile-level parallelism for the 3D FDTD method. In Proceedings of the International Conference on High Performance Computing in Asia-Pacific Region. ACM, 116-126.

[9] Van Emden Henson and Ulrike Meier Yang. 2002. BoomerAMG: A parallel algebraic multigrid solver and preconditioner. Applied Numerical Mathematics 41, 1 (2002), 155 - 177. Developments and Trends in Iterative Methods for Large Systems of Equations - in memorium Rudiger Weiss.

[10] Takeshi Iwashita, Hiroshi Nakashima, and Yasuhito Takahashi. 2012. Algebraic block multi-color ordering method for parallel multi-threaded sparse triangular solver in ICCG method. In Parallel \& Distributed Processing Symposium (IPDPS). IEEE, 474-483.

[11] George Karypis and Vipin Kumar. 1998. Multilevelk-way partitioning scheme for irregular graphs. Fournal of Parallel and Distributed computing 48, 1 (1998), 96-129.

[12] Masatoshi. Kawai, Takeshi. Iwashita, Hiroshi. Nakashima, and Osni Marques. 2013. Parallel Smoother Based on Block Red-Black Ordering for Multigrid Poisson Solver. High Performance Computing for Computational Science - VECPAR 2012 (2013).

[13] Takeshi Mifune, Takeshi Iwashita, and Masaaki Shimasaki. 2005. A parallel algebraic multigrid solver for fast magnetic edge-element analyses. IEEE transactions on magnetics 41, 5 (2005), 1660-1663.
[14] Takeshi Minami, Motoharu Hibino, Tasuku Hiraishi, Takeshi Iwashita, and Hiroshi Nakashima. 2014. Automatic parameter tuning of three-dimensional tiled fdtd kernel. In International Conference on High Performance Computing for Computational Science. Springer, 284-297.

[15] Yousef Saad. 2003. Iterative Methods for Sparse Linear Systems (2nd ed.). SIAM, Philadelphia, PA.

[16] Yonghong Song and Zhiyuan Li. 1999. New tiling techniques to improve cache temporal locality. ACM SIGPLAN Notices 34, 5 (1999), 215-228.

[17] Kosuke Takahashi, Akihiro Fujii, and Teruo Tanaka. 2011. GPGPU-based algebraic multigrid method. In Proc. 23rd IASTED International Conference on Parallel and Distributed Computing and Systems (PDCS 2011), Track. 757-061.

[18] Petr Vaněk, Jan Mandel, and Marian Brezina. 1996. Algebraic multigrid by smoothed aggregation for second and fourth order elliptic problems. Computing 56, 3 (1996), 179-196.

[19] Ulrike Meier Yang. 2006. Parallel algebraic multigrid methods-high performance preconditioners. In Numerical solution of partial differential equations on parallel computers. Springer, 209-236. 